\title{
Accurate heats of formation for polycyclic aromatic hydrocarbons: A high-level ab initio perspective
}

\author{
Amir Karton ${ }^{\mathrm{a},{ }^{*}}$ and Bun Chan ${ }^{\mathrm{b}}$

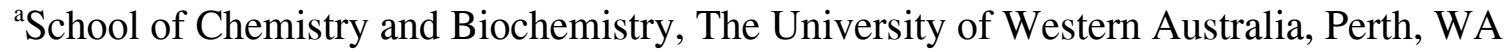 \\ 6009, Australia \\ ${ }^{\mathrm{b}}$ Graduate School of Engineering, Nagasaki University, Nagasaki, Japan

\section{Supplementary Data}

(Tables S1-S9)

\section{Total 32 Pages}

*E-Mail: amir.karton@uwa.edu.au 
Table S1. \%TAE[(T)] diagnostics for the importance of post-CCSD(T) correlation effects for the species considered in the present study (shown in Fig. 1 of the main text) ${ }^{a, b}$

\begin{tabular}{ll}
\hline Benzene & 2.0 \\
Toluene & 1.9 \\
Indene & 2.1 \\
Indane & 1.9 \\
Naphthalene & 2.2 \\
Acenaphthylene & 2.3 \\
Biphenylene & 2.4 \\
Acenaphthene & 2.1 \\
Biphenyl & 2.1 \\
Fluorene & 2.2 \\
Diphenylmethane & 2.0 \\
Pyracyclene & 2.5 \\
Anthracene & 2.3 \\
Phenanthrene & 2.3 \\
Pyracene & 2.1 \\
Pyrene & 2.4 \\
11H-Benzo[b]fluorene & 2.3 \\
Chrysene & 2.3 \\
$m$-Terphenyl & 2.2 \\
$p$-Terphenyl & 2.2 \\
\hline
\end{tabular}

${ }^{a_{0}} \% \mathrm{TAE}[(\mathrm{T})]$ is the percentages of the valence $\operatorname{CCSD}(\mathrm{T})$ atomization energy accounted for by the (T) component (for further details see refs. 1, 2, 3). ${ }^{b} \% \mathrm{TAE}[(\mathrm{T})]$ values are obtained at the CBS limit from W1-F12 theory. 
Table S2. Diagonal Born-Oppenheimer corrections for the species considered in the present study.

\begin{tabular}{lccc}
\hline & HF/cc-pVTZ & $\Delta$ CCSD/cc-pVDZ & CCSD $^{b}$ \\
\hline Benzene & 1.0 & -0.5 & 0.5 \\
Toluene & 1.2 & -0.6 & 0.6 \\
Indene & 1.5 & -0.7 & 0.8 \\
Indane & 1.5 & -0.7 & 0.8 \\
Naphthalene & 1.5 & -0.7 & 0.8 \\
Acenaphthylene & 1.7 & -0.8 & 1.0 \\
Biphenylene & 1.7 & -0.7 & 1.0 \\
Acenaphthene & 1.8 & -0.8 & 1.0 \\
Biphenyl & 1.8 & -0.8 & 1.0 \\
Fluorene & 1.9 & -0.8 & 1.1 \\
Diphenylmethane & 2.1 & -1.0 & 1.1 \\
Pyracyclene & 2.0 & -0.8 & 1.2 \\
Anthracene & 2.0 & -0.9 & 1.1 \\
Phenanthrene & 2.0 & -0.9 & 1.1 \\
Pyracene & 2.2 & -1.0 & 1.2 \\
Pyrene & 2.2 & -1.0 & 1.3 \\
Benzo[b]fluorene & 2.5 & -1.2 & 1.2 \\
Chrysene & 2.6 & -1.1 & 1.4 \\
$m$-Terphenyl & 2.7 & -1.4 & 1.4 \\
$p$-Terphenyl & 2.7 & -1.2 & 1.5 \\
\hline VDZ $=$ DBOC[CCSD/cc-pVDZ] - DBOC[HF/cc-pVDZ]. & \\
${ }^{a} \Delta$ CCSD/cc-pVD $=$ DBOC[HF/cc-pVTZ] $+\Delta C C S D / c c-p V D Z$. & &
\end{tabular}


Table S3. Stoichiometric coefficients for reactions (1) and (2) of the main text. ${ }^{a}$

\begin{tabular}{|c|c|c|c|c|c|}
\hline \multirow[t]{2}{*}{$\mathrm{C}_{n} \mathrm{H}_{m}$} & \multirow[t]{2}{*}{ Name } & \multicolumn{2}{|c|}{ Reac. (1) } & \multicolumn{2}{|c|}{ Reac. (2) } \\
\hline & & $\mathbf{a}$ & $\mathbf{b}$ & $\mathbf{a}$ & $\mathbf{b}$ \\
\hline $\mathrm{C}_{7} \mathrm{H}_{8}$ & Toluene & 1 1/9 & $1 / 3$ & 1 & $1 / 2$ \\
\hline $\mathrm{C}_{9} \mathrm{H}_{8}$ & Indene & $15 / 9$ & $-1 / 3$ & $12 / 3$ & $-1 / 2$ \\
\hline $\mathrm{C}_{9} \mathrm{H}_{10}$ & Indane & $14 / 9$ & $1 / 3$ & $11 / 3$ & $1 / 2$ \\
\hline $\mathrm{C}_{10} \mathrm{H}_{8}$ & Naphthalene & $17 / 9$ & $-2 / 3$ & 2 & -1 \\
\hline $\mathrm{C}_{12} \mathrm{H}_{8}$ & Acenaphthylene & $22 / 9$ & $-11 / 3$ & $22 / 3$ & -2 \\
\hline $\mathrm{C}_{12} \mathrm{H}_{8}$ & Biphenylene & $22 / 9$ & $-11 / 3$ & $22 / 3$ & -2 \\
\hline $\mathrm{C}_{12} \mathrm{H}_{10}$ & Acenaphthene & $21 / 9$ & $-2 / 3$ & $21 / 3$ & -1 \\
\hline $\mathrm{C}_{12} \mathrm{H}_{10}$ & Biphenyl & $21 / 9$ & $-2 / 3$ & $21 / 3$ & -1 \\
\hline $\mathrm{C}_{13} \mathrm{H}_{10}$ & Fluorene & $21 / 3$ & -1 & $22 / 3$ & $-11 / 2$ \\
\hline $\mathrm{C}_{13} \mathrm{H}_{12}$ & Diphenylmethane & $22 / 9$ & $-1 / 3$ & $21 / 3$ & $-1 / 2$ \\
\hline $\mathrm{C}_{14} \mathrm{H}_{8}$ & Pyracyclene & $22 / 3$ & -2 & $31 / 3$ & -3 \\
\hline $\mathrm{C}_{14} \mathrm{H}_{10}$ & Anthracene & $25 / 9$ & $-11 / 3$ & 3 & -2 \\
\hline $\mathrm{C}_{14} \mathrm{H}_{10}$ & Phenanthrene & $25 / 9$ & $-11 / 3$ & 3 & -2 \\
\hline $\mathrm{C}_{14} \mathrm{H}_{12}$ & Pyracene & $24 / 9$ & $-2 / 3$ & $22 / 3$ & -1 \\
\hline $\mathrm{C}_{16} \mathrm{H}_{10}$ & Pyrene & 3 & -2 & $32 / 3$ & -3 \\
\hline $\mathrm{C}_{17} \mathrm{H}_{12}$ & 11H-Benzo[b]fluorene & $31 / 9$ & $-12 / 3$ & $32 / 3$ & $-21 / 2$ \\
\hline $\mathrm{C}_{18} \mathrm{H}_{12}$ & Chrysene & $31 / 3$ & -2 & 4 & -3 \\
\hline $\mathrm{C}_{18} \mathrm{H}_{14}$ & $m$-Terphenyl & $32 / 9$ & $-11 / 3$ & $32 / 3$ & -2 \\
\hline
\end{tabular}

${ }^{a}(1) \mathrm{C}_{n} \mathrm{H}_{m} \rightarrow a \mathrm{C}_{6} \mathrm{H}_{6}+b \mathrm{CH}_{4}$; (2) $\mathrm{C}_{n} \mathrm{H}_{m} \rightarrow a \mathrm{C}_{6} \mathrm{H}_{6}+b \mathrm{C}_{2} \mathrm{H}_{4}$. 
Table S4. W1-F12 heats of formation at $0 \mathrm{~K}\left(\Delta_{f} H^{\mathrm{o}}{ }_{0}, \mathrm{~kJ} \mathrm{~mol}^{-1}\right)$ obtained from the TAE 0 values in Table 3 of the main text. ${ }^{a}$

\begin{tabular}{lc}
\hline & $\Delta_{\boldsymbol{f}} \boldsymbol{H}_{\mathbf{0}}$ \\
\hline Benzene & 100.5 \\
Toluene & 73.2 \\
Indene & 183.0 \\
Indane & 89.4 \\
Naphthalene & 170.8 \\
Acenaphthylene & 283.0 \\
Biphenylene & 438.3 \\
Acenaphthene & 183.2 \\
Biphenyl & 206.9 \\
Fluorene & 214.2 \\
Diphenylmethane & 198.7 \\
Pyracyclene & 450.8 \\
Anthracene & 255.6 \\
Phenanthrene & 231.6 \\
Pyracene & 226.1 \\
Pyrene & 250.7 \\
Benzo[b]fluorene & 282.5 \\
Chrysene & 298.3 \\
$m$-Terphenyl & 311.7 \\
$p$-Terphenyl & 311.7 \\
\hline
\end{tabular}

${ }^{a}$ The W1-F12 TAE $_{0}$ values in Table 3 were converted to heats of formation at $0 \mathrm{~K}$ using the following atomic heats of formation at $0 \mathrm{~K}$ from ATcT: ${ }^{4,5,6} \Delta_{f} H^{\mathrm{o}}{ }_{0}(\mathrm{H})=216.034 \pm$ 0.000 and $\Delta_{f} H^{\mathrm{o}} 0(\mathrm{C})=711.380 \pm 0.060 \mathrm{~kJ} \mathrm{~mol}^{-1}$. 
Table S5. W1-F12 heats of formation at $0 \mathrm{~K}\left(\Delta_{f} H^{\mathrm{o}} 0, \mathrm{~kJ} \mathrm{~mol}^{-1}\right)$ obtained from the W1-F12 TAE 0 values in Table 3 of the main text. ${ }^{a}$

\begin{tabular}{ccc}
\hline & TAE $_{0}$ & $\Delta_{f} \boldsymbol{H}^{\mathbf{0}}{ }^{298}$ \\
\hline $\mathrm{CH}_{4}$ & 1641.6 & -74.0 \\
$\mathrm{C}_{2} \mathrm{H}_{4}$ & 2225.2 & 53.1 \\
\hline
\end{tabular}


Table S6. B3LYP-D3/aug-cc-pVTZ optimized geometries ( $\AA$ ) for the species considered in the present study.

\begin{tabular}{lrrr} 
C6H6_Benzene_D6h & \\
\hline C & 0.000000 & 1.391243 & 0.000000 \\
C & 1.204852 & 0.695622 & 0.000000 \\
C & 1.204852 & -0.695622 & 0.000000 \\
$\mathrm{C}$ & 0.000000 & -1.391243 & 0.000000 \\
$\mathrm{C}$ & -1.204852 & -0.695622 & 0.000000 \\
$\mathrm{C}$ & -1.204852 & 0.695622 & 0.000000 \\
$\mathrm{H}$ & 0.000000 & 2.473155 & 0.000000 \\
$\mathrm{H}$ & 2.141815 & 1.236578 & 0.000000 \\
$\mathrm{H}$ & 2.141815 & -1.236578 & 0.000000 \\
$\mathrm{H}$ & 0.000000 & -2.473155 & 0.000000 \\
$\mathrm{H}$ & -2.141815 & -1.236578 & 0.000000 \\
$\mathrm{H}$ & -2.141815 & 1.236578 & 0.000000
\end{tabular}

\begin{tabular}{lrrr} 
C7H8_Toluene_Cs & \\
\hline $\mathrm{C}$ & 0.004969 & -0.910503 & 0.000000 \\
$\mathrm{C}$ & 0.007645 & -0.193911 & 1.197507 \\
$\mathrm{C}$ & 0.007645 & 1.196309 & 1.200488 \\
$\mathrm{C}$ & 0.006599 & 1.897701 & 0.000000 \\
$\mathrm{C}$ & 0.007645 & 1.196309 & -1.200488 \\
$\mathrm{C}$ & 0.007645 & -0.193911 & -1.197507 \\
$\mathrm{C}$ & -0.029178 & -2.416369 & 0.000000 \\
$\mathrm{H}$ & 0.012327 & -0.730827 & 2.138193 \\
$\mathrm{H}$ & 0.011984 & 1.731563 & 2.140879 \\
$\mathrm{H}$ & 0.009410 & 2.979319 & 0.000000 \\
$\mathrm{H}$ & 0.011984 & 1.731563 & -2.140879 \\
$\mathrm{H}$ & 0.012327 & -0.730827 & -2.138193 \\
$\mathrm{H}$ & 0.461715 & -2.826389 & 0.882721 \\
$\mathrm{H}$ & -1.059287 & -2.781765 & 0.000000 \\
$\mathrm{H}$ & 0.461715 & -2.826389 & -0.882721
\end{tabular}

C9H8_Indene_Cs

$\begin{array}{lrrr}\mathrm{C} & 0.000000 & 0.723782 & 0.000000 \\ \mathrm{C} & 0.427540 & -0.615907 & 0.000000 \\ \mathrm{C} & -0.499026 & -1.652728 & 0.000000 \\ \mathrm{C} & -1.855284 & -1.336555 & 0.000000 \\ \mathrm{C} & -2.277254 & -0.008456 & 0.000000 \\ \mathrm{C} & -1.347437 & 1.033067 & 0.000000 \\ \mathrm{C} & 1.213051 & 1.617827 & 0.000000\end{array}$




$\begin{array}{lrrc}\mathrm{C} & 2.352447 & 0.631293 & 0.000000 \\ \mathrm{C} & 1.889211 & -0.627818 & 0.000000 \\ \mathrm{H} & -0.175107 & -2.685392 & 0.000000 \\ \mathrm{H} & -2.590698 & -2.130118 & 0.000000 \\ \mathrm{H} & -3.335205 & 0.217213 & 0.000000 \\ \mathrm{H} & -1.682266 & 2.062753 & 0.000000 \\ \mathrm{H} & 3.391091 & 0.925632 & 0.000000 \\ \mathrm{H} & 2.490906 & -1.525291 & 0.000000 \\ \mathrm{H} & 1.240895 & 2.274091 & 0.875786 \\ \mathrm{H} & 1.240895 & 2.274091 & -0.875786\end{array}$

\begin{tabular}{lrrr} 
C9H10_Indane_Cs & & \\
\hline $\mathrm{C}$ & 0.052823 & 0.148981 & 0.697963 \\
$\mathrm{C}$ & 0.052823 & 0.148981 & -0.697963 \\
$\mathrm{C}$ & 0.004633 & -1.046311 & -1.401054 \\
$\mathrm{C}$ & -0.047822 & -2.247241 & -0.696309 \\
$\mathrm{C}$ & -0.047822 & -2.247241 & 0.696309 \\
$\mathrm{C}$ & 0.004633 & -1.046311 & 1.401054 \\
$\mathrm{C}$ & 0.130985 & 1.561151 & 1.225102 \\
$\mathrm{C}$ & -0.271296 & 2.413068 & 0.000000 \\
$\mathrm{C}$ & 0.130985 & 1.561151 & -1.225102 \\
$\mathrm{H}$ & 0.001435 & -1.049180 & -2.483890 \\
$\mathrm{H}$ & -0.094452 & -3.185329 & -1.233364 \\
$\mathrm{H}$ & -0.094452 & -3.185329 & 1.233364 \\
$\mathrm{H}$ & 0.001435 & -1.049180 & 2.483890 \\
$\mathrm{H}$ & 1.156180 & 1.787508 & 1.536650 \\
$\mathrm{H}$ & -0.509261 & 1.732017 & 2.091121 \\
$\mathrm{H}$ & 0.186719 & 3.401330 & 0.000000 \\
$\mathrm{H}$ & -1.354175 & 2.551264 & 0.000000 \\
$\mathrm{H}$ & 1.156180 & 1.787508 & -1.536650 \\
$\mathrm{H}$ & -0.509261 & 1.732017 & -2.091121
\end{tabular}

\begin{tabular}{lrrr} 
C10H8_Naphthalene_D2h & \\
\hline C & 0.000000 & 2.423179 & 0.705920 \\
C & 0.000000 & 1.240048 & 1.397230 \\
C & 0.000000 & 0.000000 & 0.714090 \\
C & 0.000000 & 0.000000 & -0.714090 \\
C & 0.000000 & 1.240048 & -1.397230 \\
C & 0.000000 & 2.423179 & -0.705920 \\
H & 0.000000 & -1.238354 & 2.480015 \\
H & 0.000000 & 3.363735 & 1.240095 \\
H & 0.000000 & 1.238354 & 2.480015 \\
C & 0.000000 & -1.240048 & 1.397230 \\
C & 0.000000 & -1.240048 & -1.397230
\end{tabular}




$\begin{array}{lrrr}\mathrm{H} & 0.000000 & 1.238354 & -2.480015 \\ \mathrm{H} & 0.000000 & 3.363735 & -1.240095 \\ \mathrm{C} & 0.000000 & -2.423179 & -0.705920 \\ \mathrm{C} & 0.000000 & -2.423179 & 0.705920 \\ \mathrm{H} & 0.000000 & -1.238354 & -2.480015 \\ \mathrm{H} & 0.000000 & -3.363735 & -1.240095 \\ \mathrm{H} & 0.000000 & -3.363735 & 1.240095\end{array}$

C12H8_Acenaphthylene_C2v

$\begin{array}{lrrr}\mathrm{C} & 0.000000 & 2.380483 & -0.320621 \\ \mathrm{C} & 0.000000 & 1.156898 & -0.947442 \\ \mathrm{C} & 0.000000 & 0.000000 & -0.139514 \\ \mathrm{C} & 0.000000 & 0.000000 & 1.251066 \\ \mathrm{C} & 0.000000 & 1.277617 & 1.873192 \\ \mathrm{C} & 0.000000 & 2.419283 & 1.099827 \\ \mathrm{H} & 0.000000 & 3.308126 & -0.878468 \\ \mathrm{C} & 0.000000 & -1.156898 & -0.947442 \\ \mathrm{C} & 0.000000 & -1.277617 & 1.873192 \\ \mathrm{H} & 0.000000 & 1.353627 & 2.952950 \\ \mathrm{H} & 0.000000 & 3.384580 & 1.588313 \\ \mathrm{C} & 0.000000 & -2.419283 & 1.099827 \\ \mathrm{C} & 0.000000 & -2.380483 & -0.320621 \\ \mathrm{H} & 0.000000 & -1.353627 & 2.952950 \\ \mathrm{H} & 0.000000 & -3.384580 & 1.588313 \\ \mathrm{H} & 0.000000 & -3.308126 & -0.878468 \\ \mathrm{C} & 0.000000 & 0.679229 & -2.336330 \\ \mathrm{C} & 0.000000 & -0.679229 & -2.336330 \\ \mathrm{H} & 0.000000 & -1.313641 & -3.209205 \\ \mathrm{H} & 0.000000 & 1.313641 & -3.209205\end{array}$

C12H8_Biphenylene_D2h

$\begin{array}{rrrr}\mathrm{C} & 0.000000 & 0.692027 & 3.110451 \\ \mathrm{C} & 0.000000 & -0.692027 & 3.110451 \\ \mathrm{C} & 0.000000 & -1.440197 & 1.908244 \\ \mathrm{C} & 0.000000 & -0.709724 & 0.752855 \\ \mathrm{C} & 0.000000 & 0.709724 & 0.752855 \\ \mathrm{C} & 0.000000 & 1.440197 & 1.908244 \\ \mathrm{H} & 0.000000 & 1.217324 & 4.056075 \\ \mathrm{H} & 0.000000 & -1.217324 & 4.056075 \\ \mathrm{H} & 0.000000 & -2.521209 & 1.927715 \\ \mathrm{H} & 0.000000 & 2.521209 & 1.927715 \\ \mathrm{C} & 0.000000 & -0.709724 & -0.752855 \\ \mathrm{C} & 0.000000 & -1.440197 & -1.908244 \\ \mathrm{C} & 0.000000 & -0.692027 & -3.110451\end{array}$




$\begin{array}{lrrr}\mathrm{C} & 0.000000 & 0.692027 & -3.110451 \\ \mathrm{C} & 0.000000 & 1.440197 & -1.908244 \\ \mathrm{C} & 0.000000 & 0.709724 & -0.752855 \\ \mathrm{H} & 0.000000 & -2.521209 & -1.927715 \\ \mathrm{H} & 0.000000 & -1.217324 & -4.056075 \\ \mathrm{H} & 0.000000 & 1.217324 & -4.056075 \\ \mathrm{H} & 0.000000 & 2.521209 & -1.927715\end{array}$

C12H10_Acenaphthene_C2v

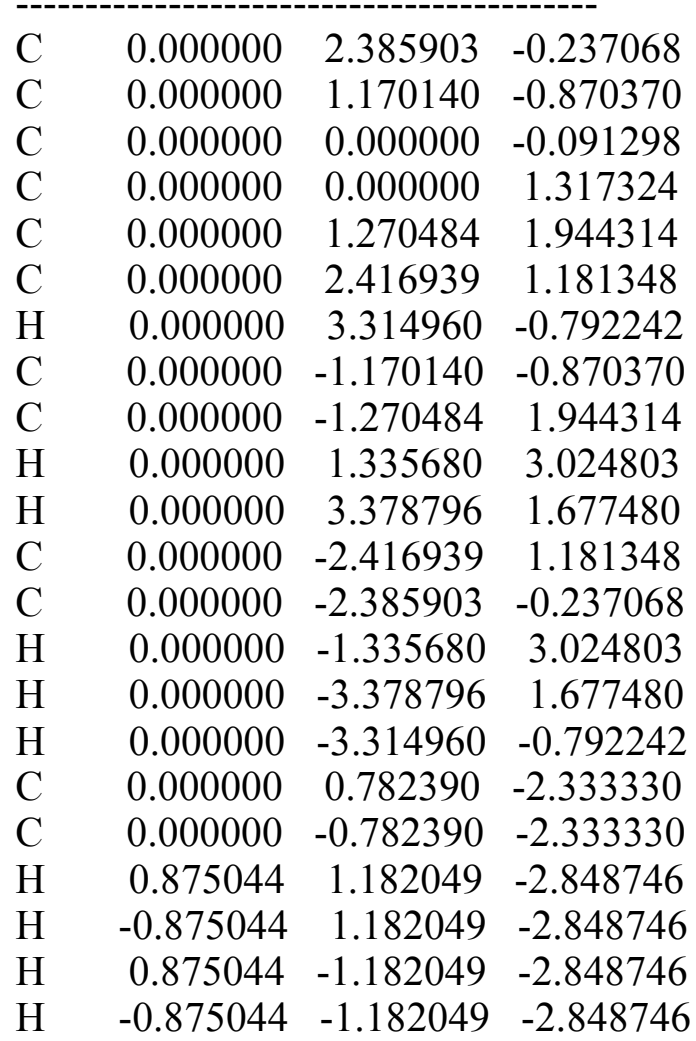

C12H10_Biphenyl_D2

$\begin{array}{lrrr}\mathrm{C} & 0.000000 & 0.000000 & -0.740493 \\ \mathrm{C} & -0.412172 & 1.127246 & -1.459633 \\ \mathrm{C} & -0.412684 & 1.127653 & -2.848340 \\ \mathrm{C} & 0.000000 & 0.000000 & -3.549535 \\ \mathrm{C} & 0.412684 & -1.127653 & -2.848340 \\ \mathrm{C} & 0.412172 & -1.127246 & -1.459633 \\ \mathrm{C} & 0.000000 & 0.000000 & 0.740493 \\ \mathrm{C} & 0.412172 & 1.127246 & 1.459633 \\ \mathrm{C} & 0.412684 & 1.127653 & 2.848340 \\ \mathrm{C} & 0.000000 & 0.000000 & 3.549535 \\ \mathrm{C} & -0.412684 & -1.127653 & 2.848340\end{array}$




$\begin{array}{rrrr}\mathrm{C} & -0.412172 & -1.127246 & 1.459633 \\ \mathrm{H} & -0.757509 & 2.002086 & -0.925277 \\ \mathrm{H} & -0.744100 & 2.007576 & -3.383599 \\ \mathrm{H} & 0.000000 & 0.000000 & -4.631134 \\ \mathrm{H} & 0.744100 & -2.007576 & -3.383599 \\ \mathrm{H} & 0.757509 & -2.002086 & -0.925277 \\ \mathrm{H} & 0.757509 & 2.002086 & 0.925277 \\ \mathrm{H} & 0.744100 & 2.007576 & 3.383599 \\ \mathrm{H} & 0.000000 & 0.000000 & 4.631134 \\ \mathrm{H} & -0.744100 & -2.007576 & 3.383599 \\ \mathrm{H} & -0.757509 & -2.002086 & 0.925277\end{array}$

C13H10_Fluorene_C2v

$\begin{array}{lrrr}\mathrm{H} & -0.877183 & 0.000000 & 2.478523 \\ \mathrm{C} & 0.000000 & 3.003272 & -1.207715 \\ \mathrm{C} & 0.000000 & 3.447123 & 0.113997 \\ \mathrm{C} & 0.000000 & 2.534643 & 1.168335 \\ \mathrm{C} & 0.000000 & 1.179969 & 0.883487 \\ \mathrm{C} & 0.000000 & 0.731786 & -0.447854 \\ \mathrm{C} & 0.000000 & 1.643077 & -1.498726 \\ \mathrm{H} & 0.000000 & 3.724767 & -2.013778 \\ \mathrm{H} & 0.000000 & 4.508549 & 0.322509 \\ \mathrm{H} & 0.000000 & 2.885178 & 2.192696 \\ \mathrm{H} & 0.000000 & 1.302784 & -2.525917 \\ \mathrm{C} & 0.000000 & 0.000000 & 1.825606 \\ \mathrm{H} & 0.877183 & 0.000000 & 2.478523 \\ \mathrm{C} & 0.000000 & -1.179969 & 0.883487 \\ \mathrm{C} & 0.000000 & -2.534643 & 1.168335 \\ \mathrm{C} & 0.000000 & -3.447123 & 0.113997 \\ \mathrm{C} & 0.000000 & -3.003272 & -1.207715 \\ \mathrm{C} & 0.000000 & -1.643077 & -1.498726 \\ \mathrm{C} & 0.000000 & -0.731786 & -0.447854 \\ \mathrm{H} & 0.000000 & -2.885178 & 2.192696 \\ \mathrm{H} & 0.000000 & -4.508549 & 0.322509 \\ \mathrm{H} & 0.000000 & -3.724767 & -2.013778 \\ \mathrm{H} & 0.000000 & -1.302784 & -2.525917\end{array}$

C13H12_Diphenylmethane_C2

$\begin{array}{lrrr}\mathrm{C} & 0.000000 & 0.000000 & 1.481281 \\ \mathrm{C} & 0.000000 & 1.266542 & 0.651139 \\ \mathrm{C} & -1.035490 & 2.193190 & 0.747213 \\ \mathrm{C} & 1.045714 & 1.526305 & -0.237199 \\ \mathrm{C} & -1.029417 & 3.354502 & -0.020726 \\ \mathrm{H} & -1.855591 & 2.006678 & 1.429591\end{array}$




$\begin{array}{lrrr}\mathrm{C} & 1.057896 & 2.683683 & -1.003006 \\ \mathrm{H} & 1.850352 & 0.808212 & -0.333912 \\ \mathrm{C} & 0.018023 & 3.603740 & -0.897771 \\ \mathrm{H} & -1.843656 & 4.061568 & 0.067685 \\ \mathrm{H} & 1.877293 & 2.868578 & -1.685043 \\ \mathrm{H} & 0.025747 & 4.504950 & -1.495898 \\ \mathrm{C} & 0.000000 & -1.266542 & 0.651139 \\ \mathrm{C} & 1.035490 & -2.193190 & 0.747213 \\ \mathrm{C} & -1.045714 & -1.526305 & -0.237199 \\ \mathrm{C} & 1.029417 & -3.354502 & -0.020726 \\ \mathrm{C} & -1.057896 & -2.683683 & -1.003006 \\ \mathrm{C} & -0.018023 & -3.603740 & -0.897771 \\ \mathrm{H} & -0.025747 & -4.504950 & -1.495898 \\ \mathrm{H} & -1.850352 & -0.808212 & -0.333912 \\ \mathrm{H} & -1.877293 & -2.868578 & -1.685043 \\ \mathrm{H} & 1.843656 & -4.061568 & 0.067685 \\ \mathrm{H} & 1.855591 & -2.006678 & 1.429591 \\ \mathrm{H} & 0.874397 & -0.002839 & 2.135837 \\ \mathrm{H} & -0.874397 & 0.002839 & 2.135837\end{array}$

C14H8_Pyracyclene_D2h

\begin{tabular}{lrrr}
\hline C & 0.000000 & 0.680508 & 2.838437 \\
$\mathrm{C}$ & 0.000000 & 1.176683 & 1.435186 \\
$\mathrm{C}$ & 0.000000 & 0.000000 & 0.674129 \\
$\mathrm{C}$ & 0.000000 & -1.176683 & 1.435186 \\
$\mathrm{C}$ & 0.000000 & -0.680508 & 2.838437 \\
$\mathrm{C}$ & 0.000000 & 2.362726 & 0.723143 \\
$\mathrm{C}$ & 0.000000 & 0.000000 & -0.674129 \\
$\mathrm{C}$ & 0.000000 & 1.176683 & -1.435186 \\
$\mathrm{C}$ & 0.000000 & 2.362726 & -0.723143 \\
$\mathrm{C}$ & 0.000000 & 0.680508 & -2.838437 \\
$\mathrm{H}$ & 0.000000 & 1.300404 & -3.721817 \\
$\mathrm{C}$ & 0.000000 & -0.680508 & -2.838437 \\
$\mathrm{C}$ & 0.000000 & -1.176683 & -1.435186 \\
$\mathrm{C}$ & 0.000000 & -2.362726 & -0.723143 \\
$\mathrm{C}$ & 0.000000 & -2.362726 & 0.723143 \\
$\mathrm{H}$ & 0.000000 & -3.321904 & 1.224951 \\
$\mathrm{H}$ & 0.000000 & -3.321904 & -1.224951 \\
$\mathrm{H}$ & 0.000000 & 3.321904 & 1.224951 \\
$\mathrm{H}$ & 0.000000 & 1.300404 & 3.721817 \\
$\mathrm{H}$ & 0.000000 & -1.300404 & 3.721817 \\
$\mathrm{H}$ & 0.000000 & 3.321904 & -1.224951 \\
$\mathrm{H}$ & 0.000000 & -1.300404 & -3.721817
\end{tabular}

C14H10_Anthracene_D2h 


$\begin{array}{lrrr}\text { C } & 0.000000 & 3.645788 & 0.710921 \\ \mathrm{C} & 0.000000 & 2.470209 & 1.401990 \\ \mathrm{C} & 0.000000 & 1.218525 & 0.720015 \\ \mathrm{C} & 0.000000 & 1.218525 & -0.720015 \\ \mathrm{C} & 0.000000 & 2.470209 & -1.401990 \\ \mathrm{C} & 0.000000 & 3.645788 & -0.710921 \\ \mathrm{C} & 0.000000 & 0.000000 & 1.398762 \\ \mathrm{C} & 0.000000 & 0.000000 & -1.398762 \\ \mathrm{C} & 0.000000 & -1.218525 & -0.720015 \\ \mathrm{C} & 0.000000 & -1.218525 & 0.720015 \\ \mathrm{C} & 0.000000 & -2.470209 & 1.401990 \\ \mathrm{H} & 0.000000 & -2.469506 & 2.484764 \\ \mathrm{C} & 0.000000 & -3.645788 & 0.710921 \\ \mathrm{C} & 0.000000 & -3.645788 & -0.710921 \\ \mathrm{C} & 0.000000 & -2.470209 & -1.401990 \\ \mathrm{H} & 0.000000 & 0.000000 & 2.482447 \\ \mathrm{H} & 0.000000 & 4.588327 & 1.241781 \\ \mathrm{H} & 0.000000 & 2.469506 & 2.484764 \\ \mathrm{H} & 0.000000 & 2.469506 & -2.484764 \\ \mathrm{H} & 0.000000 & 4.588327 & -1.241781 \\ \mathrm{H} & 0.000000 & 0.000000 & -2.482447 \\ \mathrm{H} & 0.000000 & -4.588327 & 1.241781 \\ \mathrm{H} & 0.000000 & -4.588327 & -1.241781 \\ \mathrm{H} & 0.000000 & -2.469506 & -2.484764 \\ & & & \end{array}$

\begin{tabular}{lrrr} 
C14H10_Phenanthrene_C2v & \\
\hline C & 0.000000 & 3.548203 & -0.294929 \\
C & 0.000000 & 2.827076 & 0.875552 \\
C & 0.000000 & 1.417442 & 0.862473 \\
C & 0.000000 & 0.726341 & -0.379480 \\
C & 0.000000 & 1.495264 & -1.561054 \\
C & 0.000000 & 2.871989 & -1.523558 \\
C & 0.000000 & 0.676696 & 2.086317 \\
C & 0.000000 & -0.726341 & -0.379480 \\
C & 0.000000 & -1.417442 & 0.862473 \\
C & 0.000000 & -0.676696 & 2.086317 \\
C & 0.000000 & -2.827076 & 0.875552 \\
H & 0.000000 & -3.337148 & 1.830660 \\
C & 0.000000 & -3.548203 & -0.294929 \\
C & 0.000000 & -2.871989 & -1.523558 \\
C & 0.000000 & -1.495264 & -1.561054 \\
H & 0.000000 & 1.225517 & 3.019564 \\
H & 0.000000 & 4.629513 & -0.270770 \\
H & 0.000000 & 3.337148 & 1.830660 \\
H & 0.000000 & 1.005564 & -2.523364
\end{tabular}




$\begin{array}{lrrr}\mathrm{H} & 0.000000 & 3.433731 & -2.448009 \\ \mathrm{H} & 0.000000 & -1.225517 & 3.019564 \\ \mathrm{H} & 0.000000 & -4.629513 & -0.270770 \\ \mathrm{H} & 0.000000 & -3.433731 & -2.448009 \\ \mathrm{H} & 0.000000 & -1.005564 & -2.523364\end{array}$

C14H12_Pyracene_D2h

\begin{tabular}{|c|c|c|c|}
\hline $\mathrm{C}$ & 2.894203 & 0.790775 & 0.000000 \\
\hline & 1.425827 & 1.186603 & 0.000000 \\
\hline 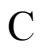 & 0.688597 & 0.000000 & 0.000000 \\
\hline $\mathrm{C}$ & 1.425827 & -1.186603 & 0.000000 \\
\hline $\mathrm{C}$ & 2.894203 & -0.790775 & 0.000000 \\
\hline $\mathrm{C}$ & 0.717595 & 2.370123 & 0.000000 \\
\hline $\mathrm{C}$ & -0.688597 & 0.000000 & 0.000000 \\
\hline $\mathrm{C}$ & -1.425827 & 1.186603 & 0.000000 \\
\hline $\mathrm{C}$ & -0.717595 & 2.370123 & 0.000000 \\
\hline $\mathrm{C}$ & -2.894203 & 0.790775 & 0.000000 \\
\hline $\mathrm{H}$ & -3.415663 & 1.182015 & 0.875232 \\
\hline $\mathrm{C}$ & -2.894203 & -0.790775 & 0.000000 \\
\hline $\mathrm{C}$ & -1.425827 & -1.186603 & 0.000000 \\
\hline $\mathrm{C}$ & -0.717595 & -2.370123 & 0.000000 \\
\hline $\mathrm{C}$ & 0.717595 & -2.370123 & 0.000000 \\
\hline $\mathrm{H}$ & 1.227353 & -3.325479 & 0.000000 \\
\hline $\mathrm{H}$ & -1.227353 & -3.325479 & 0.000000 \\
\hline $\mathrm{H}$ & 1.227353 & 3.325479 & 0.000000 \\
\hline $\mathrm{H}$ & 3.415663 & 1.182015 & 0.875232 \\
\hline $\mathrm{H}$ & 3.415663 & -1.182015 & 0.875232 \\
\hline $\mathrm{H}$ & -1.227353 & 3.325479 & 0.000000 \\
\hline $\mathrm{H}$ & -3.415663 & -1.182015 & -0.875232 \\
\hline $\mathrm{H}$ & -3.415663 & -1.182015 & 0.875232 \\
\hline $\mathrm{H}$ & -3.415663 & 1.182015 & -0.875232 \\
\hline $\mathrm{H}$ & 3.415663 & 1.182015 & -0.875232 \\
\hline $\mathrm{H}$ & 3.415663 & -1.182015 & -0.875232 \\
\hline
\end{tabular}

C16H10_Pyrene_D2h

$\begin{array}{lrrr}\mathrm{C} & 0.000000 & 0.000000 & 3.509845 \\ \mathrm{C} & 0.000000 & 1.206133 & 2.822000 \\ \mathrm{C} & 0.000000 & 1.231493 & 1.423448 \\ \mathrm{C} & 0.000000 & 0.000000 & 0.710606 \\ \mathrm{C} & 0.000000 & -1.231493 & 1.423448 \\ \mathrm{C} & 0.000000 & -1.206133 & 2.822000 \\ \mathrm{C} & 0.000000 & 2.455206 & 0.677694 \\ \mathrm{C} & 0.000000 & 0.000000 & -0.710606 \\ \mathrm{C} & 0.000000 & 1.231493 & -1.423448\end{array}$




$\begin{array}{lrrr}\mathrm{C} & 0.000000 & 2.455206 & -0.677694 \\ \mathrm{C} & 0.000000 & 1.206133 & -2.822000 \\ \mathrm{H} & 0.000000 & 2.141491 & -3.366924 \\ \mathrm{C} & 0.000000 & 0.000000 & -3.509845 \\ \mathrm{C} & 0.000000 & -1.206133 & -2.822000 \\ \mathrm{C} & 0.000000 & -1.231493 & -1.423448 \\ \mathrm{C} & 0.000000 & -2.455206 & -0.677694 \\ \mathrm{C} & 0.000000 & -2.455206 & 0.677694 \\ \mathrm{H} & 0.000000 & -3.389849 & 1.224208 \\ \mathrm{H} & 0.000000 & -3.389849 & -1.224208 \\ \mathrm{H} & 0.000000 & 3.389849 & 1.224208 \\ \mathrm{H} & 0.000000 & 0.000000 & 4.591623 \\ \mathrm{H} & 0.000000 & 2.141491 & 3.366924 \\ \mathrm{H} & 0.000000 & -2.141491 & 3.366924 \\ \mathrm{H} & 0.000000 & 3.389849 & -1.224208 \\ \mathrm{H} & 0.000000 & 0.000000 & -4.591623 \\ \mathrm{H} & 0.000000 & -2.141491 & -3.366924\end{array}$

\begin{tabular}{lrrl} 
C17H12_11H-Benzo-b-fluorene Cs \\
\hline--1.990233 & -0.021369 & 0.877238 \\
H & -2.919136 & -1.311213 & 0.000000 \\
$\mathrm{C}$ & 0.555172 & -2.270104 & 0.000000 \\
$\mathrm{C}$ & -0.790423 & -1.816944 & 0.000000 \\
$\mathrm{C}$ & -1.058761 & -0.479703 & 0.000000 \\
$\mathrm{C}$ & 0.000000 & 0.475223 & 0.000000 \\
$\mathrm{C}$ & 1.309904 & 0.070157 & 0.000000 \\
$\mathrm{H}$ & -1.590194 & -2.548050 & 0.000000 \\
$\mathrm{H}$ & 2.117434 & 0.791753 & 0.000000 \\
$\mathrm{C}$ & -2.390303 & 0.235301 & 0.000000 \\
$\mathrm{H}$ & -2.990233 & -0.021369 & -0.877238 \\
$\mathrm{C}$ & -1.989743 & 1.691918 & 0.000000 \\
$\mathrm{C}$ & -2.791905 & 2.820770 & 0.000000 \\
$\mathrm{C}$ & -2.190400 & 4.078086 & 0.000000 \\
$\mathrm{C}$ & -0.800972 & 4.199638 & 0.000000 \\
$\mathrm{C}$ & 0.008158 & 3.069259 & 0.000000 \\
$\mathrm{C}$ & -0.590883 & 1.813163 & 0.000000 \\
$\mathrm{H}$ & -3.870948 & 2.732650 & 0.000000 \\
$\mathrm{H}$ & -2.805627 & 4.967803 & 0.000000 \\
$\mathrm{H}$ & -0.350350 & 5.183071 & 0.000000 \\
$\mathrm{H}$ & 1.085809 & 3.166976 & 0.000000 \\
$\mathrm{C}$ & 0.880346 & -3.647085 & 0.000000 \\
$\mathrm{H}$ & 0.075120 & -4.371210 & 0.000000 \\
$\mathrm{C}$ & 2.956011 & -1.780049 & 0.000000 \\
$\mathrm{H}$ & 3.760422 & -1.055110 & 0.000000 \\
$\mathrm{C}$ & 3.235074 & -3.122104 & 0.000000 \\
$\mathrm{C}$ & 2.186263 & -4.066226 & 0.000000
\end{tabular}




$\begin{array}{llll}\mathrm{H} & 2.416936 & -5.123045 & 0.000000 \\ \mathrm{H} & 4.261821 & -3.462620 & 0.000000\end{array}$

C18H12_Chrysene_C2h

\begin{tabular}{lrrl}
\hline $\mathrm{C}$ & 2.433561 & 0.718355 & 0.000000 \\
$\mathrm{C}$ & 1.253087 & 1.388888 & 0.000000 \\
$\mathrm{C}$ & 0.000871 & 0.705905 & 0.000000 \\
$\mathrm{C}$ & -0.000871 & -0.705905 & 0.000000 \\
$\mathrm{C}$ & 1.253087 & -1.429844 & 0.000000 \\
$\mathrm{C}$ & 2.475256 & -0.702900 & 0.000000 \\
$\mathrm{C}$ & -1.253087 & 1.429844 & 0.000000 \\
$\mathrm{C}$ & -1.253087 & -1.388888 & 0.000000 \\
$\mathrm{C}$ & -2.433561 & -0.718355 & 0.000000 \\
$\mathrm{C}$ & -2.475256 & 0.702900 & 0.000000 \\
$\mathrm{C}$ & -3.705461 & 1.394656 & 0.000000 \\
$\mathrm{H}$ & -4.623137 & 0.820005 & 0.000000 \\
$\mathrm{C}$ & -3.745186 & 2.766713 & 0.000000 \\
$\mathrm{C}$ & -2.543059 & 3.493216 & 0.000000 \\
$\mathrm{C}$ & -1.332210 & 2.840958 & 0.000000 \\
$\mathrm{H}$ & 1.273534 & 2.467518 & 0.000000 \\
$\mathrm{H}$ & -1.273534 & -2.467518 & 0.000000 \\
$\mathrm{H}$ & -4.693136 & 3.287431 & 0.000000 \\
$\mathrm{H}$ & -2.568507 & 4.574669 & 0.000000 \\
$\mathrm{H}$ & -0.431147 & 3.434756 & 0.000000 \\
$\mathrm{C}$ & 3.705461 & -1.394656 & 0.000000 \\
$\mathrm{H}$ & 4.623137 & -0.820005 & 0.000000 \\
$\mathrm{C}$ & 1.332210 & -2.840958 & 0.000000 \\
$\mathrm{H}$ & 0.431147 & -3.434756 & 0.000000 \\
$\mathrm{C}$ & 3.745186 & -2.766713 & 0.000000 \\
$\mathrm{C}$ & 2.543059 & -3.493216 & 0.000000 \\
$\mathrm{H}$ & 4.693136 & -3.287431 & 0.000000 \\
$\mathrm{H}$ & 2.568507 & -4.574669 & 0.000000 \\
$\mathrm{H}$ & 3.368325 & 1.264575 & 0.000000 \\
$\mathrm{H}$ & -3.368325 & -1.264575 & 0.000000
\end{tabular}

C18H14_m-Terphenyl_C2

$\begin{array}{lrrr}\mathrm{C} & -0.004828 & 1.221052 & 0.750272 \\ \mathrm{C} & -0.005746 & 1.205285 & 2.148576 \\ \mathrm{C} & 0.000000 & 0.000000 & 2.837060 \\ \mathrm{C} & 0.005746 & -1.205285 & 2.148576 \\ \mathrm{C} & 0.004828 & -1.221052 & 0.750272 \\ \mathrm{C} & 0.000000 & 0.000000 & 0.072560 \\ \mathrm{C} & 0.000000 & 2.500209 & 0.002995 \\ \mathrm{C} & -0.778339 & 3.582319 & 0.427964\end{array}$




$\begin{array}{lrrr}\mathrm{C} & -0.776253 & 4.781778 & -0.271933 \\ \mathrm{C} & 0.005765 & 4.925956 & -1.412632 \\ \mathrm{C} & 0.785103 & 3.859164 & -1.846374 \\ \mathrm{C} & 0.781706 & 2.659808 & -1.146324 \\ \mathrm{H} & 0.013697 & 2.137973 & 2.695530 \\ \mathrm{H} & 0.000000 & 0.000000 & 3.919009 \\ \mathrm{H} & -0.013697 & -2.137973 & 2.695530 \\ \mathrm{H} & 0.000000 & 0.000000 & -1.008880 \\ \mathrm{H} & -1.407954 & 3.474325 & 1.300945 \\ \mathrm{H} & -1.392934 & 5.602450 & 0.069818 \\ \mathrm{H} & 0.007979 & 5.860101 & -1.957815 \\ \mathrm{H} & 1.404272 & 3.963134 & -2.727503 \\ \mathrm{H} & 1.409829 & 1.844860 & -1.480162 \\ \mathrm{C} & 0.000000 & -2.500209 & 0.002995 \\ \mathrm{C} & -0.781706 & -2.659808 & -1.146324 \\ \mathrm{C} & 0.778339 & -3.582319 & 0.427964 \\ \mathrm{C} & -0.785103 & -3.859164 & -1.846374 \\ \mathrm{H} & -1.409829 & -1.844860 & -1.480162 \\ \mathrm{C} & 0.776253 & -4.781778 & -0.271933 \\ \mathrm{H} & 1.407954 & -3.474325 & 1.300945 \\ \mathrm{C} & -0.005765 & -4.925956 & -1.412632 \\ \mathrm{H} & -1.404272 & -3.963134 & -2.727503 \\ \mathrm{H} & 1.392934 & -5.602450 & 0.069818 \\ \mathrm{H} & -0.007979 & -5.860101 & -1.957815\end{array}$

C18H14_p-Terphenyl_D2

\begin{tabular}{rrrr}
\hline $\mathrm{C}$ & 0.000000 & 0.000000 & -2.897404 \\
$\mathrm{C}$ & -0.761913 & 0.927467 & -3.617032 \\
$\mathrm{C}$ & -0.762400 & 0.927654 & -5.005621 \\
$\mathrm{C}$ & 0.000000 & 0.000000 & -5.706945 \\
$\mathrm{C}$ & 0.762400 & -0.927654 & -5.005621 \\
$\mathrm{C}$ & 0.761913 & -0.927467 & -3.617032 \\
$\mathrm{C}$ & 0.000000 & 0.000000 & -1.417788 \\
$\mathrm{C}$ & -0.000730 & 1.196166 & -0.693007 \\
$\mathrm{C}$ & 0.000730 & 1.196166 & 0.693007 \\
$\mathrm{C}$ & 0.000000 & 0.000000 & 1.417788 \\
$\mathrm{C}$ & -0.000730 & -1.196166 & 0.693007 \\
$\mathrm{C}$ & 0.000730 & -1.196166 & -0.693007 \\
$\mathrm{H}$ & -1.377866 & 1.638668 & -3.083270 \\
$\mathrm{H}$ & -1.366342 & 1.648368 & -5.540806 \\
$\mathrm{H}$ & 0.000000 & 0.000000 & -6.788529 \\
$\mathrm{H}$ & 1.366342 & -1.648368 & -5.540806 \\
$\mathrm{H}$ & 1.377866 & -1.638668 & -3.083270 \\
$\mathrm{H}$ & 0.013518 & 2.139842 & -1.221596 \\
$\mathrm{H}$ & -0.013518 & 2.139842 & 1.221596 \\
$\mathrm{H}$ & 0.013518 & -2.139842 & 1.221596
\end{tabular}




$\begin{array}{lrcc}\mathrm{H} & -0.013518 & -2.139842 & -1.221596 \\ \mathrm{C} & 0.000000 & 0.000000 & 2.897404 \\ \mathrm{C} & -0.761913 & -0.927467 & 3.617032 \\ \mathrm{C} & 0.761913 & 0.927467 & 3.617032 \\ \mathrm{C} & -0.762400 & -0.927654 & 5.005621 \\ \mathrm{H} & -1.377866 & -1.638668 & 3.083270 \\ \mathrm{C} & 0.762400 & 0.927654 & 5.005621 \\ \mathrm{H} & 1.377866 & 1.638668 & 3.083270 \\ \mathrm{C} & 0.000000 & 0.000000 & 5.706945 \\ \mathrm{H} & -1.366342 & -1.648368 & 5.540806 \\ \mathrm{H} & 1.366342 & 1.648368 & 5.540806 \\ \mathrm{H} & 0.000000 & 0.000000 & 6.788529\end{array}$


Table S7. B3LYP-D3/aug-cc-pVTZ harmonic frequencies $\left(\mathrm{cm}^{-1}\right)$ for the species considered in the present study.

\begin{tabular}{lcc} 
C6H6_Benzene_D6h & \\
\hline------------ & \\
413.8048 & 413.8048 & 622.8986 \\
622.8986 & 691.9988 & 722.3806 \\
869.5481 & 869.5481 & 999.5095 \\
999.5095 & 1012.4049 & 1026.7887 \\
1027.5300 & 1061.4708 & 1061.4708 \\
1183.3415 & 1204.2992 & 1204.2992 \\
1334.4300 & 1385.9322 & 1515.1258 \\
1515.1258 & 1632.7922 & 1632.7922 \\
3154.7350 & 3165.4485 & 3165.4485 \\
3181.9929 & 3181.9929 & 3192.1996 \\
& & \\
\hline----------------- & \\
C7H8_Toluene_Cs & \\
------------------ & \\
21.0386 & 208.0642 & 354.7688 \\
416.8011 & 477.6233 & 531.1421 \\
637.4612 & 715.0309 & 748.2173 \\
800.9657 & 863.0601 & 921.0028 \\
997.3465 & 1008.4226 & 1015.3547 \\
1021.3699 & 1054.5740 & 1066.4770 \\
1116.4199 & 1189.3946 & 1212.8807 \\
1230.2440 & 1327.8684 & 1363.5563 \\
1416.8693 & 1470.9146 & 1492.9992 \\
1503.7899 & 1532.5722 & 1623.3118 \\
1645.3562 & 3019.5575 & 3072.2566 \\
3097.9966 & 3151.6946 & 3153.4106 \\
3166.8027 & 3175.5444 & 3188.4055 \\
20.3969 & 208.0481 & 354.7644 \\
416.8021 & 477.6137 & 531.1386 \\
637.4612 & 715.0322 & 748.2161 \\
800.9645 & 863.0602 & 921.0011 \\
997.3470 & 1008.4159 & 1015.3555 \\
1021.3691 & 1054.5732 & 1066.4679 \\
1116.4187 & 1189.3949 & 1212.8791 \\
1230.2432 & 1327.8682 & 1363.5543 \\
1416.8541 & 1470.9106 & 1492.9938 \\
1503.7855 & 1532.5703 & 1623.3114 \\
1645.3545 & 3019.5519 & 3072.2562 \\
3098.0058 & 3151.6920 & 3153.4069 \\
3166.8016 & 3175.5457 & 3188.4065
\end{tabular}


C9H8_Indene_Cs

\begin{tabular}{lcl}
\hline 194.5519 & 212.2223 & 387.8123 \\
399.2409 & 429.3487 & 542.3664 \\
567.5910 & 605.6640 & 710.9989 \\
738.5031 & 746.3687 & 789.8860 \\
846.0884 & 875.7028 & 878.8928 \\
944.4877 & 952.8386 & 965.1910 \\
978.5715 & 1007.0258 & 1042.2945 \\
1091.2071 & 1136.3159 & 1148.1690 \\
1183.0382 & 1190.2270 & 1228.3075 \\
1250.2706 & 1316.3774 & 1347.1257 \\
1392.2750 & 1434.4537 & 1490.2737 \\
1493.8272 & 1599.3150 & 1633.3287 \\
1648.1335 & 3013.6419 & 3035.0412 \\
3156.1814 & 3162.8856 & 3174.1459 \\
3186.6816 & 3189.0785 & 3213.2238
\end{tabular}

$\begin{array}{lll}\text { C9H10_Indane_Cs } & \\ ----- & \\ 146.6159 & 183.1319 & 257.2464 \\ 380.0259 & 427.9565 & 514.1541 \\ 524.1577 & 594.8187 & 628.6518 \\ 724.7070 & 754.0457 & 769.3788 \\ 844.0868 & 869.4512 & 880.2389 \\ 913.6715 & 916.4981 & 961.4665 \\ 1003.5577 & 1008.7076 & 1046.7568 \\ 1065.5310 & 1107.7779 & 1155.5483 \\ 1180.4639 & 1186.7369 & 1200.8079 \\ 1226.1488 & 1245.6002 & 1295.0748 \\ 1300.7678 & 1333.5946 & 1344.8412 \\ 1358.1018 & 1483.7540 & 1484.3039 \\ 1493.0623 & 1507.7046 & 1513.4308 \\ 1623.2047 & 1645.6819 & 3000.5303 \\ 3002.5198 & 3034.4372 & 3063.0709 \\ 3065.7991 & 3087.9813 & 3153.3286 \\ 3158.8221 & 3171.0242 & 3184.9655\end{array}$

C10H8_Naphthalene_D2h

$\begin{array}{lll}171.2429 & 183.1451 & 372.0982 \\ 396.4719 & 480.9112 & 489.1791 \\ 517.6613 & 521.4090 & 634.6643 \\ 635.4576 & 733.4224 & 776.0656 \\ 791.3105 & 805.1611 & 810.8538 \\ 854.5150 & 906.8231 & 955.7781\end{array}$




$\begin{array}{lcc}972.6254 & 993.5450 & 1007.3162 \\ 1012.4248 & 1036.8474 & 1051.8456 \\ 1158.4635 & 1176.4033 & 1177.8255 \\ 1197.3711 & 1235.9347 & 1275.8853 \\ 1298.2251 & 1394.9721 & 1396.7910 \\ 1423.7172 & 1493.6169 & 1497.3296 \\ 1549.3900 & 1611.9865 & 1640.1843 \\ 1669.2117 & 3155.0720 & 3156.7734 \\ 3159.1130 & 3162.3956 & 3174.3412 \\ 3175.5015 & 3187.8413 & 3188.7440\end{array}$

C12H8_Acenaphthylene_C2v

\begin{tabular}{lll}
\hline 158.0860 & 211.9130 & 228.0232 \\
377.4607 & 423.7286 & 465.2731 \\
469.4296 & 518.2362 & 562.0913 \\
586.8132 & 621.4999 & 671.0702 \\
680.3393 & 697.2812 & 749.9417 \\
773.3629 & 796.3605 & 820.2069 \\
867.0055 & 882.0494 & 930.4926 \\
945.1208 & 949.3619 & 999.6992 \\
1005.9270 & 1031.0044 & 1036.0929 \\
1057.6515 & 1102.7780 & 1117.2830 \\
1181.2640 & 1210.7370 & 1227.2556 \\
1249.8062 & 1275.8165 & 1334.4139 \\
1387.2695 & 1421.0028 & 1450.7038 \\
1457.4236 & 1489.6350 & 1516.6120 \\
1537.8883 & 1636.4230 & 1650.5559 \\
1659.4833 & 3159.6604 & 3159.9275 \\
3169.2054 & 3169.8588 & 3183.6725 \\
3184.4408 & 3202.3651 & 3221.9885
\end{tabular}

C12H8_Biphenylene_D2h

$\begin{array}{llc}101.0458 & 145.2226 & 211.5578 \\ 318.6166 & 379.0963 & 398.8526 \\ 432.8315 & 467.8430 & 570.0327 \\ 592.4244 & 615.7119 & 628.6884 \\ 732.5877 & 735.5777 & 752.3156 \\ 763.6626 & 782.6122 & 824.4573 \\ 891.0935 & 906.5748 & 945.8531 \\ 950.4209 & 988.3022 & 1000.3235 \\ 1001.6211 & 1011.9267 & 1012.5298 \\ 1045.0639 & 1077.4211 & 1112.4754 \\ 1129.0010 & 1149.2803 & 1185.6609 \\ 1197.8508 & 1292.9143 & 1299.9117\end{array}$




$\begin{array}{lll}1316.1585 & 1433.0754 & 1460.2405 \\ 1478.8261 & 1482.0522 & 1499.7044 \\ 1622.1326 & 1628.7089 & 1642.6104 \\ 1702.8817 & 3163.1822 & 3163.5695 \\ 3175.5201 & 3176.2824 & 3184.6620 \\ 3185.4712 & 3191.2665 & 3192.1212\end{array}$

C12H10_Acenaphthene_C2v

\begin{tabular}{lcc}
\hline 80.9468 & 161.6238 & 218.0864 \\
234.1418 & 424.9429 & 450.6348 \\
454.2012 & 477.6543 & 510.5401 \\
553.8121 & 569.9772 & 636.1349 \\
647.4281 & 667.0487 & 765.9112 \\
772.4321 & 806.1401 & 818.4047 \\
840.5406 & 869.6545 & 900.9455 \\
929.4436 & 944.6825 & 993.9447 \\
1002.8338 & 1017.4522 & 1029.4205 \\
1039.6576 & 1067.1126 & 1111.3710 \\
1173.7411 & 1182.7630 & 1205.1082 \\
1237.9713 & 1242.5833 & 1248.1937 \\
1252.5834 & 1283.5977 & 1309.6761 \\
1389.3560 & 1394.7939 & 1451.5471 \\
1466.7032 & 1470.2462 & 1495.5477 \\
1504.2039 & 1532.8498 & 1634.8691 \\
1638.9459 & 1658.0430 & 3029.7698 \\
3039.9750 & 3050.6494 & 3071.4582 \\
3155.8941 & 3156.0611 & 3166.7828 \\
3167.5085 & 3180.1420 & 3181.1032
\end{tabular}

C12H10_Biphenyl_D2

$\begin{array}{lcc}71.2703 & 93.8267 & 129.9504 \\ 273.4295 & 316.6785 & 371.0997 \\ 416.2321 & 424.3137 & 506.3901 \\ 561.8333 & 626.6424 & 629.2660 \\ 641.4785 & 718.3317 & 720.4617 \\ 758.3219 & 761.1040 & 804.1236 \\ 864.8358 & 865.8198 & 934.1420 \\ 953.5232 & 1000.8237 & 1001.5688 \\ 1013.8467 & 1018.1014 & 1018.2211 \\ 1022.7415 & 1030.8340 & 1057.4292 \\ 1071.5165 & 1108.3166 & 1112.2864 \\ 1190.8163 & 1192.0497 & 1213.3277 \\ 1222.5285 & 1300.6289 & 1305.4008 \\ 1325.7762 & 1363.9725 & 1366.6114\end{array}$




$\begin{array}{lll}1465.0125 & 1492.9875 & 1521.0564 \\ 1542.4600 & 1608.2543 & 1623.2282 \\ 1641.4341 & 1643.6045 & 3158.7345 \\ 3159.7889 & 3164.0628 & 3167.1516 \\ 3174.0971 & 3177.9091 & 3180.6863 \\ 3183.3584 & 3190.2193 & 3191.3351\end{array}$

C13H10_Fluorene_C2v

$\begin{array}{lcc}97.4191 & 137.5255 & 218.4201 \\ 242.9166 & 276.3903 & 421.1586 \\ 425.0795 & 441.4057 & 485.3645 \\ 500.5211 & 553.9554 & 581.2427 \\ 636.9844 & 645.9535 & 718.6630 \\ 748.4073 & 758.9429 & 760.7494 \\ 806.6718 & 815.5287 & 856.4132 \\ 879.1635 & 892.0253 & 941.5726 \\ 969.1416 & 986.6736 & 1007.9682 \\ 1009.6561 & 1027.4702 & 1046.8603 \\ 1054.7606 & 1121.7014 & 1133.6750 \\ 1163.1176 & 1181.4545 & 1187.9979 \\ 1196.1465 & 1211.9583 & 1222.9263 \\ 1254.7694 & 1324.7531 & 1331.5371 \\ 1345.5062 & 1376.8540 & 1449.1624 \\ 1480.9563 & 1487.4408 & 1511.5423 \\ 1513.3614 & 1617.4449 & 1621.3232 \\ 1647.8292 & 1648.3728 & 3020.6389 \\ 3044.6574 & 3157.2630 & 3157.6943 \\ 3163.8004 & 3165.3524 & 3175.1344 \\ 3176.4144 & 3187.6341 & 3188.2567\end{array}$

C13H12_Diphenylmethane_C2

$\begin{array}{lcc}15.9465 & 19.7945 & 62.7411 \\ 191.1425 & 232.2085 & 290.5051 \\ 354.4523 & 416.1988 & 416.5554 \\ 465.0946 & 483.8682 & 566.0392 \\ 624.4389 & 635.3976 & 638.5809 \\ 716.9106 & 719.7364 & 757.2182 \\ 757.7193 & 834.3736 & 837.5035 \\ 863.5906 & 863.9109 & 913.6784 \\ 945.0073 & 953.9148 & 998.1354 \\ 998.3303 & 1016.7119 & 1016.8466 \\ 1022.0641 & 1022.5958 & 1053.5305 \\ 1054.2570 & 1100.9255 & 1112.7370 \\ 1188.9779 & 1189.1133 & 1196.1901\end{array}$




$\begin{array}{lll}1211.1311 & 1212.1022 & 1217.1274 \\ 1221.8736 & 1305.2443 & 1341.9070 \\ 1346.1974 & 1369.4480 & 1371.4617 \\ 1481.7592 & 1485.4030 & 1494.3113 \\ 1530.0360 & 1532.0437 & 1621.5569 \\ 1625.5513 & 1640.7333 & 1646.5956 \\ 3023.4292 & 3056.9686 & 3152.7429 \\ 3152.8825 & 3156.7109 & 3157.2485 \\ 3167.7567 & 3168.0410 & 3176.3914 \\ 3176.5809 & 3188.4110 & 3188.6230\end{array}$

C14H8_Pyracyclene_D2h

\begin{tabular}{lcc}
\hline 82.5277 & 174.7061 & 241.3027 \\
337.1418 & 369.7763 & 393.8577 \\
437.4788 & 496.3323 & 511.5188 \\
534.5649 & 544.1452 & 561.1093 \\
638.5260 & 665.4212 & 680.7923 \\
698.4562 & 707.6049 & 735.3490 \\
774.3399 & 785.6413 & 849.3107 \\
855.3997 & 865.0180 & 868.1820 \\
942.8903 & 947.0349 & 952.4836 \\
967.5428 & 971.5152 & 987.3480 \\
1051.5982 & 1054.9342 & 1090.0191 \\
1099.5148 & 1140.7712 & 1144.6063 \\
1226.3036 & 1243.1869 & 1268.9466 \\
1313.1101 & 1347.8525 & 1359.2825 \\
1419.8919 & 1437.8407 & 1475.9427 \\
1487.1159 & 1491.1478 & 1519.2293 \\
1540.4529 & 1616.5347 & 1651.9315 \\
1780.4088 & 3160.0957 & 3160.1215 \\
3175.4051 & 3175.6944 & 3201.4876 \\
3201.8741 & 3221.2646 & 3221.4635
\end{tabular}

C14H10_Anthracene_D2h

$\begin{array}{lcc}90.4797 & 118.5261 & 235.5829 \\ 239.0140 & 266.1730 & 389.6327 \\ 399.5221 & 401.3808 & 481.6416 \\ 487.2345 & 507.9217 & 538.0976 \\ 591.1824 & 617.7264 & 641.6816 \\ 662.1619 & 746.9518 & 762.9075 \\ 764.4856 & 776.3263 & 788.3260 \\ 827.8428 & 850.1879 & 874.6109 \\ 912.8989 & 923.3702 & 926.2233 \\ 933.6837 & 985.2776 & 991.0575\end{array}$




$\begin{array}{lll}1007.5599 & 1007.8486 & 1030.0313 \\ 1033.0046 & 1130.4858 & 1163.8500 \\ 1181.2778 & 1199.0864 & 1201.8807 \\ 1219.1020 & 1291.6647 & 1298.1800 \\ 1311.0831 & 1342.4380 & 1380.2577 \\ 1416.3050 & 1420.2591 & 1425.3163 \\ 1488.6003 & 1488.8340 & 1519.1094 \\ 1578.6587 & 1592.1251 & 1623.8643 \\ 1665.8250 & 1668.0973 & 3151.2147 \\ 3153.1025 & 3155.9233 & 3156.6125 \\ 3160.6219 & 3161.9099 & 3174.7493 \\ 3174.9955 & 3187.4518 & 3187.7980\end{array}$

C14H10_Phenanthrene_C2v

$\begin{array}{lcc}\text { 93.9581 } & 99.7264 & 227.8256 \\ 241.7378 & 250.7072 & 402.1735 \\ 413.1241 & 439.6731 & 449.1888 \\ 509.4759 & 510.2366 & 548.6560 \\ 557.0878 & 602.0413 & 632.1580 \\ 724.9445 & 729.1199 & 732.6929 \\ 753.9740 & 775.4725 & 811.8205 \\ 836.1788 & 845.7635 & 892.7522 \\ 892.9645 & 893.8774 & 973.7646 \\ 982.0464 & 997.4647 & 1009.3679 \\ 1011.6400 & 1020.6499 & 1064.3601 \\ 1066.3911 & 1120.8731 & 1173.1795 \\ 1180.8096 & 1196.7231 & 1200.5746 \\ 1228.2066 & 1246.7716 & 1275.9789 \\ 1312.9429 & 1328.5863 & 1365.8268 \\ 1374.0222 & 1452.4040 & 1453.8383 \\ 1474.1293 & 1496.5494 & 1537.5527 \\ 1562.9442 & 1605.8915 & 1642.2657 \\ 1654.0121 & 1658.9054 & 3154.2271 \\ 3157.8751 & 3158.5030 & 3168.1498 \\ 3169.6072 & 3174.6937 & 3182.7874 \\ 3186.0258 & 3192.9472 & 3205.3259\end{array}$

C14H12_Pyracene_D2h

$\begin{array}{lcc}90.0691 & 108.3549 & 118.4794 \\ 150.3971 & 303.7590 & 305.7121 \\ 381.4039 & 464.0866 & 467.4035 \\ 480.1497 & 502.2728 & 528.8900 \\ 533.7408 & 606.7931 & 631.9498 \\ 644.8055 & 663.8885 & 753.8981\end{array}$




$\begin{array}{lll}775.6682 & 787.2335 & 803.1141 \\ 828.6427 & 864.1901 & 868.6027 \\ 905.6778 & 911.4575 & 935.1260 \\ 941.6994 & 960.2503 & 964.3277 \\ 1023.1713 & 1027.8732 & 1075.7742 \\ 1077.6623 & 1133.2771 & 1153.9179 \\ 1167.3767 & 1171.5077 & 1230.6898 \\ 1236.5618 & 1237.2550 & 1241.7136 \\ 1242.4417 & 1256.2733 & 1282.9284 \\ 1284.1158 & 1319.0340 & 1376.3257 \\ 1395.7998 & 1465.3337 & 1470.1441 \\ 1471.2205 & 1476.9299 & 1493.7253 \\ 1496.0784 & 1516.7036 & 1531.8823 \\ 1622.6387 & 1654.6431 & 1703.5575 \\ 3028.9944 & 3029.2354 & 3039.5222 \\ 3039.7995 & 3050.6956 & 3050.7646 \\ 3071.7994 & 3071.9682 & 3152.1980 \\ 3152.7460 & 3168.7255 & 3168.9174\end{array}$

\begin{tabular}{lcc} 
C16H10_Pyrene_D2h & \\
- \hdashline--- & \\
97.5606 & 150.3046 & 212.2959 \\
248.8243 & 262.0370 & 363.1859 \\
404.5690 & 412.0441 & 462.6775 \\
503.0730 & 511.2525 & 513.6467 \\
519.5647 & 543.4582 & 553.4871 \\
591.3753 & 598.2467 & 698.5082 \\
705.2728 & 733.9731 & 752.0824 \\
762.8225 & 791.2176 & 817.8733 \\
823.6573 & 837.7316 & 871.2538 \\
874.1367 & 919.2062 & 933.1972 \\
983.0209 & 990.7080 & 999.0224 \\
999.2512 & 1005.5629 & 1019.0695 \\
1096.5035 & 1121.7903 & 1132.1275 \\
1172.1211 & 1180.4500 & 1207.7253 \\
1218.7584 & 1234.6156 & 1264.7204 \\
1271.0058 & 1273.8821 & 1344.1893 \\
1357.5458 & 1397.4189 & 1427.1615 \\
1443.3248 & 1460.7963 & 1465.8619 \\
1486.5442 & 1514.0150 & 1536.5677 \\
1595.8452 & 1623.4924 & 1634.3511 \\
1643.7118 & 1668.3900 & 3154.6906 \\
3154.9046 & 3158.0648 & 3158.5008 \\
3166.0236 & 3166.7338 & 3174.3979 \\
3174.4001 & 3183.8455 & 3184.1722
\end{tabular}


C17H12_11H-Benzo-b-fluorene_Cs

\begin{tabular}{llc}
\hline 60.9892 & 96.0398 & 155.7585 \\
157.2579 & 208.4538 & 274.8654 \\
313.8494 & 315.3235 & 360.3899 \\
408.0046 & 439.5180 & 488.1887 \\
490.2412 & 507.1660 & 552.0360 \\
570.6885 & 583.1731 & 618.2204 \\
644.2425 & 686.4535 & 715.4559 \\
746.6295 & 762.3604 & 767.9659 \\
786.6799 & 797.0846 & 807.6353 \\
845.3814 & 866.1037 & 885.3084 \\
899.8119 & 912.7803 & 920.3872 \\
957.8984 & 981.2754 & 987.8634 \\
1003.0780 & 1007.7711 & 1009.2985 \\
1046.2372 & 1048.1277 & 1121.2984 \\
1141.0063 & 1167.3632 & 1174.4840 \\
1183.0293 & 1187.3747 & 1202.7403 \\
1217.8984 & 1228.3949 & 1256.1024 \\
1264.8131 & 1292.4814 & 1330.6148 \\
1351.9272 & 1374.7700 & 1388.0890 \\
1430.4495 & 1454.1753 & 1472.3878 \\
1483.4418 & 1505.5254 & 1507.1554 \\
1540.0464 & 1614.1792 & 1621.1293 \\
1646.8683 & 1651.4292 & 1675.4198 \\
3020.7813 & 3044.4323 & 3152.4904 \\
3154.9824 & 3157.8616 & 3158.5467 \\
3161.4110 & 3165.3466 & 3173.7672 \\
3176.1795 & 3187.3812 & 3188.2936
\end{tabular}

C18H12_Chrysene_C2h

\begin{tabular}{lcc}
\hline 48.5707 & 75.7941 & 132.5234 \\
175.6590 & 189.6351 & 235.7810 \\
292.7016 & 296.2365 & 387.1062 \\
393.1823 & 440.2537 & 487.2144 \\
488.9027 & 489.4690 & 524.2201 \\
546.6703 & 568.4221 & 577.8720 \\
579.3439 & 594.5168 & 692.5109 \\
693.1171 & 697.2632 & 754.2101 \\
758.0664 & 782.4321 & 784.5878 \\
802.6584 & 837.2190 & 856.7007 \\
867.3214 & 890.8268 & 892.4269 \\
896.3337 & 897.2451 & 973.5951 \\
979.6841 & 991.2144 & 996.6876 \\
1009.6063 & 1011.1974 & 1040.9333 \\
1060.8585 & 1067.4538 & 1106.5869
\end{tabular}




$\begin{array}{lll}1168.4877 & 1180.7779 & 1185.6741 \\ 1197.3033 & 1216.8898 & 1220.3541 \\ 1252.4487 & 1264.6177 & 1284.1361 \\ 1294.4542 & 1329.0177 & 1356.4937 \\ 1380.1043 & 1389.0438 & 1394.5874 \\ 1459.6374 & 1467.2640 & 1468.2334 \\ 1491.7483 & 1523.9055 & 1556.6990 \\ 1560.9390 & 1604.3500 & 1636.7399 \\ 1646.6677 & 1656.2049 & 1658.3164 \\ 3157.6804 & 3157.8790 & 3161.9658 \\ 3162.8288 & 3169.3695 & 3169.8335 \\ 3185.2827 & 3185.6158 & 3195.8790 \\ 3196.0967 & 3213.3779 & 3213.6242\end{array}$

C18H14_m-Terphenyl_C2

\begin{tabular}{llc}
\hline 45.1085 & 48.6971 & 62.1540 \\
98.1795 & 114.7935 & 145.2208 \\
236.6525 & 279.1102 & 291.3553 \\
336.5206 & 351.6088 & 405.9619 \\
420.1567 & 420.4051 & 493.8630 \\
504.7809 & 553.4970 & 627.8038 \\
628.8765 & 632.1812 & 635.9965 \\
648.3767 & 719.0945 & 719.7131 \\
725.4613 & 728.2655 & 774.7821 \\
789.0280 & 824.8388 & 829.3946 \\
865.3678 & 865.8508 & 929.2560 \\
932.8779 & 950.4831 & 954.5264 \\
1001.2216 & 1001.5415 & 1008.6266 \\
1013.3242 & 1018.3961 & 1018.4235 \\
1020.7201 & 1022.3228 & 1033.1702 \\
1053.4776 & 1067.7355 & 1092.7203 \\
1110.5051 & 1110.5356 & 1129.3133 \\
1191.5359 & 1191.5454 & 1209.0666 \\
1218.2164 & 1219.6291 & 1270.9144 \\
1295.5114 & 1309.8884 & 1323.3412 \\
1334.3313 & 1357.4102 & 1362.2758 \\
1369.2599 & 1437.5169 & 1477.7990 \\
1490.6960 & 1515.4544 & 1533.8616 \\
1539.4049 & 1608.7701 & 1614.6369 \\
1624.1566 & 1640.0820 & 1642.7286 \\
1642.9888 & 3159.3731 & 3159.4395 \\
3162.8986 & 3165.7058 & 3165.7727 \\
3173.9388 & 3175.1837 & 3177.9408 \\
3179.0357 & 3182.4740 & 3182.7252 \\
3188.6130 & 3190.8245 & 3191.1759
\end{tabular}




\begin{tabular}{lll}
\hline C18H14_p-Terphenyl_D2 & \\
\hdashline 37.7223 & 47.8130 & 69.6940 \\
88.5676 & 123.4664 & 137.8901 \\
216.3132 & 252.8656 & 294.6684 \\
392.6235 & 410.2960 & 416.8551 \\
422.3014 & 427.7200 & 427.9909 \\
532.7369 & 533.6859 & 585.7908 \\
628.1909 & 632.2270 & 635.0238 \\
657.3833 & 714.8733 & 718.8085 \\
719.1884 & 751.1354 & 778.3256 \\
792.1361 & 797.0445 & 860.5849 \\
864.7841 & 864.9603 & 871.4093 \\
941.1616 & 947.5276 & 989.7921 \\
1000.8209 & 1001.0636 & 1002.7641 \\
1015.3674 & 1017.9030 & 1018.1200 \\
1019.1931 & 1028.0907 & 1029.9199 \\
1051.9114 & 1064.3644 & 1075.3863 \\
1109.9886 & 1110.6000 & 1154.8718 \\
1191.4684 & 1191.6855 & 1215.4786 \\
1217.9216 & 1232.7147 & 1288.1510 \\
1305.2429 & 1309.3571 & 1312.0608 \\
1322.9418 & 1350.5863 & 1363.3030 \\
1367.3506 & 1437.1936 & 1475.4871 \\
1487.1513 & 1522.6389 & 1532.3007 \\
1558.5670 & 1586.3852 & 1615.7516 \\
1620.2263 & 1640.8222 & 1642.3928 \\
1651.5054 & 3159.1449 & 3159.4008 \\
3163.4822 & 3165.3401 & 3165.5349 \\
3168.1654 & 3175.4248 & 3176.5635 \\
3180.2104 & 3182.2345 & 3184.0147 \\
3184.8382 & 3190.8033 & 3191.1027
\end{tabular}


Table S8. Chemical compounds and models considered in the present work. The structures are shown in Figure 1 of the main text and cartesian coordinates are given in Table S4.

\begin{tabular}{|c|c|c|c|c|c|}
\hline & Mol. Formula & Name & Symmetry & CAS \# & Model \\
\hline 1 & $\mathrm{C}_{6} \mathrm{H}_{6}$ & Benzene & $\mathrm{D}_{6 \mathrm{~h}}$ & $71-43-2$ & W1-F12 theory \\
\hline 2 & $\mathrm{C}_{7} \mathrm{H}_{8}$ & Toluene & $\mathrm{C}_{s}$ & $108-88-3$ & W1-F12 theory \\
\hline 3 & $\mathrm{C}_{9} \mathrm{H}_{8}$ & Indene & $\mathrm{C}_{\mathrm{s}}$ & $95-13-6$ & W1-F12 theory \\
\hline 4 & $\mathrm{C}_{9} \mathrm{H}_{10}$ & Indane & $\mathrm{C}_{\mathrm{s}}$ & $496-11-7$ & W1-F12 theory \\
\hline 5 & $\mathrm{C}_{10} \mathrm{H}_{8}$ & Naphthalene & $\mathrm{D}_{2 \mathrm{~h}}$ & $91-20-3$ & W1-F12 theory \\
\hline 6 & $\mathrm{C}_{12} \mathrm{H}_{8}$ & Acenaphthylene & $\mathrm{C}_{2 \mathrm{v}}$ & $208-96-8$ & W1-F12 theory \\
\hline 7 & $\mathrm{C}_{12} \mathrm{H}_{8}$ & Biphenylene & $\mathrm{D}_{2 \mathrm{~h}}$ & $259-79-0$ & W1-F12 theory \\
\hline 8 & $\mathrm{C}_{12} \mathrm{H}_{10}$ & Acenaphthene & $\mathrm{C}_{2 \mathrm{v}}$ & $83-32-9$ & W1-F12 theory \\
\hline 9 & $\mathrm{C}_{12} \mathrm{H}_{10}$ & Biphenyl & $\mathrm{D}_{2}$ & $92-52-4$ & W1-F12 theory \\
\hline 10 & $\mathrm{C}_{13} \mathrm{H}_{10}$ & Fluorene & $\mathrm{C}_{2 \mathrm{v}}$ & $86-73-7$ & W1-F12 theory \\
\hline 11 & $\mathrm{C}_{13} \mathrm{H}_{12}$ & Diphenylmethane & $\mathrm{C}_{2}$ & $101-81-5$ & W1-F12 theory \\
\hline 12 & $\mathrm{C}_{14} \mathrm{H}_{8}$ & Pyracyclene & $\mathrm{D}_{2 \mathrm{~h}}$ & $187-78-0$ & W1-F12 theory \\
\hline 13 & $\mathrm{C}_{14} \mathrm{H}_{10}$ & Anthracene & $\mathrm{D}_{2 \mathrm{~h}}$ & $120-12-7$ & W1-F12 theory \\
\hline 14 & $\mathrm{C}_{14} \mathrm{H}_{10}$ & Phenanthrene & $\mathrm{C}_{2 \mathrm{v}}$ & $85-01-8$ & W1-F12 theory \\
\hline 15 & $\mathrm{C}_{14} \mathrm{H}_{12}$ & Pyracene & $\mathrm{D}_{2 \mathrm{~h}}$ & $567-79-3$ & W1-F12 theory \\
\hline 16 & $\mathrm{C}_{16} \mathrm{H}_{10}$ & Pyrene & $\mathrm{D}_{2 \mathrm{~h}}$ & $129-00-0$ & W1-F12 theory \\
\hline 17 & $\mathrm{C}_{17} \mathrm{H}_{12}$ & $11 \mathrm{H}$-Benzo[b]fluorene & $\mathrm{C}_{\mathrm{s}}$ & $243-17-4$ & W1-F12 theory \\
\hline 18 & $\mathrm{C}_{18} \mathrm{H}_{12}$ & Chrysene & $\mathrm{C}_{2 \mathrm{~h}}$ & 218-01-9 & W1-F12 theory \\
\hline 19 & $\mathrm{C}_{18} \mathrm{H}_{14}$ & $m$-Terphenyl & $\mathrm{C}_{2}$ & $92-06-8$ & W1-F12 theory \\
\hline 20 & $\mathrm{C}_{18} \mathrm{H}_{14}$ & $p$-Terphenyl & $\mathrm{D}_{2}$ & $92-94-4$ & W1-F12 theory \\
\hline
\end{tabular}


Table S9. Full References for Molpro 2012, CFOUR, and Gaussian 09.

(22) MOLPRO, version 2012.1, a package of ab initio programs, Werner, H.-J.; Knowles, P. J.; Manby, F. R.; Schütz, M.; Celani, P.; Knizia, G.; Korona, T.; Lindh, R.; Mitrushenkov, A.; Rauhut, G.; Adler, T. B.; Amos, R. D.; Bernhardsson, A.; Berning, A.; Cooper, D. L.; Deegan, M. J. O.; Dobbyn, A. J.; Eckert, F.; Goll, E.; Hampel, C.; Hesselmann, A.; Hetzer, G.; Hrenar, T.; Jansen, G.; Köppl, C.; Liu, Y.; Lloyd, A. W.; Mata, R. A.; May, A. J.; McNicholas, S. J.; Meyer, W.; Mura, M. E.; Nicklaß, A.; Palmieri, P.; Pflüger, K.; Pitzer, R.; Reiher, M.; Shiozaki, T.; Stoll, H. Stone, A. J.; Tarroni, R.; Thorsteinsson, T.; Wang, M. See also: http:www.molpro.net.

(41) CFOUR, a quantum chemical program package written by J.F. Stanton, J. Gauss, M.E. Harding, P.G. Szalay with contributions from A.A. Auer, R.J. Bartlett, U. Benedikt, C. Berger, D.E. Bernholdt, Y.J. Bomble, L. Cheng, O. Christiansen, M. Heckert, O. Heun, C. Huber, T.-C. Jagau, D. Jonsson, J. Jusélius, K. Klein, W.J. Lauderdale, F. Lipparini, D.A. Matthews, T. Metzroth, L.A. Mück, D.P. O'Neill, D.R. Price, E. Prochnow, C. Puzzarini, K. Ruud, F. Schiffmann, W. Schwalbach, C. Simmons, S. Stopkowicz, A. Tajti, J. Vázquez, F. Wang, J.D. Watts and the integral packages MOLECULE (J. Almlöf and P.R. Taylor), PROPS (P.R. Taylor), ABACUS (T. Helgaker, H.J. Aa. Jensen, P. Jørgensen, and J. Olsen), and ECP routines by A. V. Mitin and C. van Wüllen. For the current version, see http://www.cfour.de.

(50) Gaussian 09, Revision D.01, Frisch, M. J.; Trucks, G. W.; Schlegel, H. B.; Scuseria, G. E.; Robb, M. A.; Cheeseman, J. R.; Scalmani, G.; Barone, V.; Mennucci, B.; Petersson, G. A.; Nakatsuji, H.; Caricato, M.; Li, X.; Hratchian, H. P.; Izmaylov, A. F.; Bloino, J.; Zheng, G.; Sonnenberg, J. L.; Hada, M.; Ehara, M.; Toyota, K.; Fukuda, R.; Hasegawa, J.; Ishida, M.; Nakajima, T.; Honda, Y.; Kitao, O.; Nakai, H.; Vreven, T.; Montgomery, Jr., J. A.; Peralta, J. E.; Ogliaro, F.; Bearpark, M.; Heyd, J. J.; Brothers, E.; Kudin, K. N.; Staroverov, V. N.; Kobayashi, R.; Normand, J.; Raghavachari, K.; Rendell, A.; Burant, J. C.; Iyengar, S. S.; Tomasi, J.; Cossi, M.; Rega, N.; Millam, N. J.; Klene, M.; Knox, J. E.; Cross, J. B.; Bakken, V.; Adamo, C.; Jaramillo, J.; Gomperts, R.; Stratmann, R. E.; Yazyev, O.; Austin, A. J.; Cammi, R.; Pomelli, C.; Ochterski, J. W.; Martin, R. L.; Morokuma, K.; Zakrzewski, V. G.; Voth, G. A.; Salvador, P.; Dannenberg, J. J.; Dapprich, S.; Daniels, A. D.; Farkas, Ö.; Foresman, J. B.; Ortiz, J. V.; Cioslowski, J.; Fox, D. J. Gaussian, Inc., Wallingford CT, 2009. 


\section{References}

${ }^{1}$ Karton, A.; Rabinovich, E.; Martin, J. M. L.; Ruscic, B. J. Chem. Phys. 2006, 125, 144108.

${ }^{2}$ Karton, A.; Daon, S.; Martin, J. M. L. Chem. Phys. Lett. 2011, 510, 165.

${ }^{3}$ Karton, A. Wiley Interdiscip. Rev. Comput. Mol. Sci. 2016, 6, 292.

${ }^{4}$ Ruscic, B.; Pinzon, R. E.; Morton, M. L.; von Laszewski, G.; Bittner, S.; Nijsure, S. G.;

Amin, K. A.; Minkoff, M.; Wagner, A. F. J. Phys. Chem. A 2004, 108, 9979.

${ }^{5}$ Ruscic, B.; Pinzon, R. E.; Morton, M. L.; Srinivasan, N. K.; Su, M. C.; Sutherland, J. W.; Michael, J. V. J. Phys. Chem. A 2006, 110, 6592.

${ }^{6}$ Stevens, W. R.; Ruscic, B.; Baer, T. J. Phys. Chem. A 2010, 114, 13134. 